\title{
Scheme for Universal High-Dimensional Quantum Computation with Linear Optics
}

\author{
Stefano Paesani $\odot,{ }^{1,2, *}$ Jacob F. F. Bulmer $\odot,{ }^{1}$ Alex E. Jones $\odot,{ }^{1}$ Raffaele Santagati, ${ }^{1,3}$ and Anthony Laing ${ }^{1}$ \\ ${ }^{1}$ Quantum Engineering Technology Labs, H. H. Wills Physics Laboratory and Department of Electrical and Electronic Engineering, \\ University of Bristol, Bristol BS8 1FD, United Kingdom \\ ${ }^{2}$ Center for Hybrid Quantum Networks (Hy-Q), Niels Bohr Institute, University of Copenhagen, \\ Blegdamsvej 17, DK-2100 Copenhagen, Denmark \\ ${ }^{3}$ International Iberian Nanotechnology Laboratory (INL), Avenida Mestre José Veiga 4715-330 Braga, Portugal
}

(Received 25 October 2020; accepted 19 March 2021; published 11 June 2021)

\begin{abstract}
Photons are natural carriers of high-dimensional quantum information, and, in principle, can benefit from higher quantum information capacity and noise resilience. However, schemes to generate the resources required for high-dimensional quantum computing have so far been lacking in linear optics. Here, we show how to generate GHZ states in arbitrary dimensions and numbers of photons using linear optical circuits described by Fourier transform matrices. Combining our results with recent schemes for qudit Bell measurements, we show that universal linear optical quantum computing can be performed in arbitrary dimensions.
\end{abstract}

DOI: 10.1103/PhysRevLett.126.230504

Photonics is a sophisticated platform for the development of quantum technologies, from quantum processors to distributed quantum communication [1-5]. Until now, linear optical architectures have focused on encoding photons as qubits (two-level systems). Yet using higher dimensional systems - qudits - can in principle improve the information capacity and noise tolerance of computational resources, and potentially unlock new routes to fault-tolerant quantum computing and distributed quantum networks [6]. Qudits can naturally be encoded in photons using $d$ orthogonal optical modes and in a variety of degrees of freedom, e.g., spatial modes [7-9], orbital angular-momentum [10-14], optical frequencies [15,16], and time bins [17,18]. Highprecision control and arbitrary operations of single qudits have been demonstrated using programmable interferometers $[7,8,19,20]$. However, architectures for universal quantum photonic processors based on higher-dimensional systems have, so far, been absent. While there has been significant experimental progress in photonic qudit entanglement generation [7-16,18], the postselected schemes used so far can only generate a limited set of highdimensional entangled states and present no clear route to scalability $[21,22]$. In fact, in contrast to the qubit case $[23,24]$, even determining which high-dimensional entangled states can be generated with single photons and linear optics has so far remained an open problem [6].

Published by the American Physical Society under the terms of the Creative Commons Attribution 4.0 International license. Further distribution of this work must maintain attribution to the author(s) and the published article's title, journal citation, and DOI.
Here, we answer this question by showing that all highdimensional entangled states with fixed numbers of photons can be generated with linear optics and, in principle, with a scalable architecture. That is, we show that universal linear optical quantum computing (LOQC) is possible in arbitrary dimensions. Key to this result are linear optical schemes for the generation of heralded $N$-photon GHZ entanglement in arbitrary dimension $d$. Combining these schemes with previous results on Bell measurements with photonic qudits to fuse $d$-dimensional GHZ states, and techniques from qubit LOQC architectures, we obtain a scheme for universal measurement-based quantum computing with photonic qudits.

Heralded high-dimensional GHZ entanglement.The GHZ states for $N$ photons, each encoding a qudit of dimension $d$, are defined as $|\operatorname{GHZ}(N, d)\rangle=$ $\sum_{k=0}^{d-1}|k\rangle^{\otimes N} / \sqrt{d}$, and represent the initial resource states in an architecture that builds cluster states of qudits for universal quantum computing. Our scheme to generate these states, shown schematically in Fig. 1(a), consists of single photon sources, linear optical elements, and photonnumber resolving detectors. Our scheme exploits the zerotransmission law (ZTL) in discrete Fourier transform (DFT) interferometers (previously investigated, e.g., for the verification of boson sampling protocols $[19,25,26])$. This law is a generalization of the Hong-Ou-Mandel interference that suppresses output coincidences from a balanced beam splitter (two-mode DFT). The ZTL result states that, if $m$ single photons are sent in the $m$ individual input modes of an $m$-mode DFT, described by the unitary $U_{j, k}=\exp [j k(2 \pi \mathrm{i} / m)] / \sqrt{m}$, then the output configurations $\vec{c}$ with nonzero amplitude must satisfy 
(a)

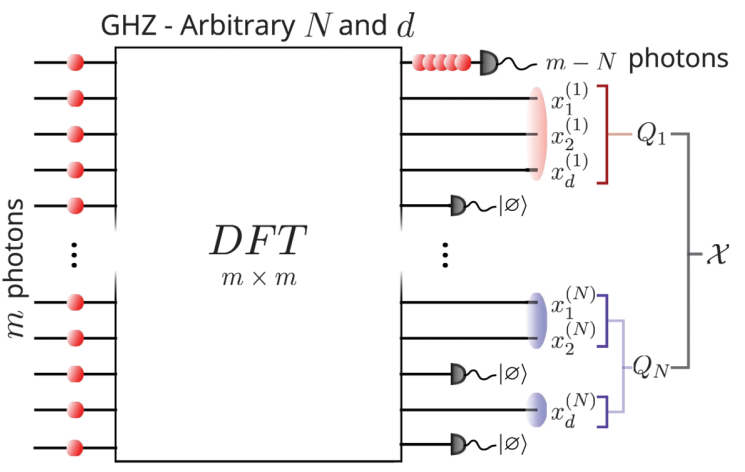

(b)

(c)
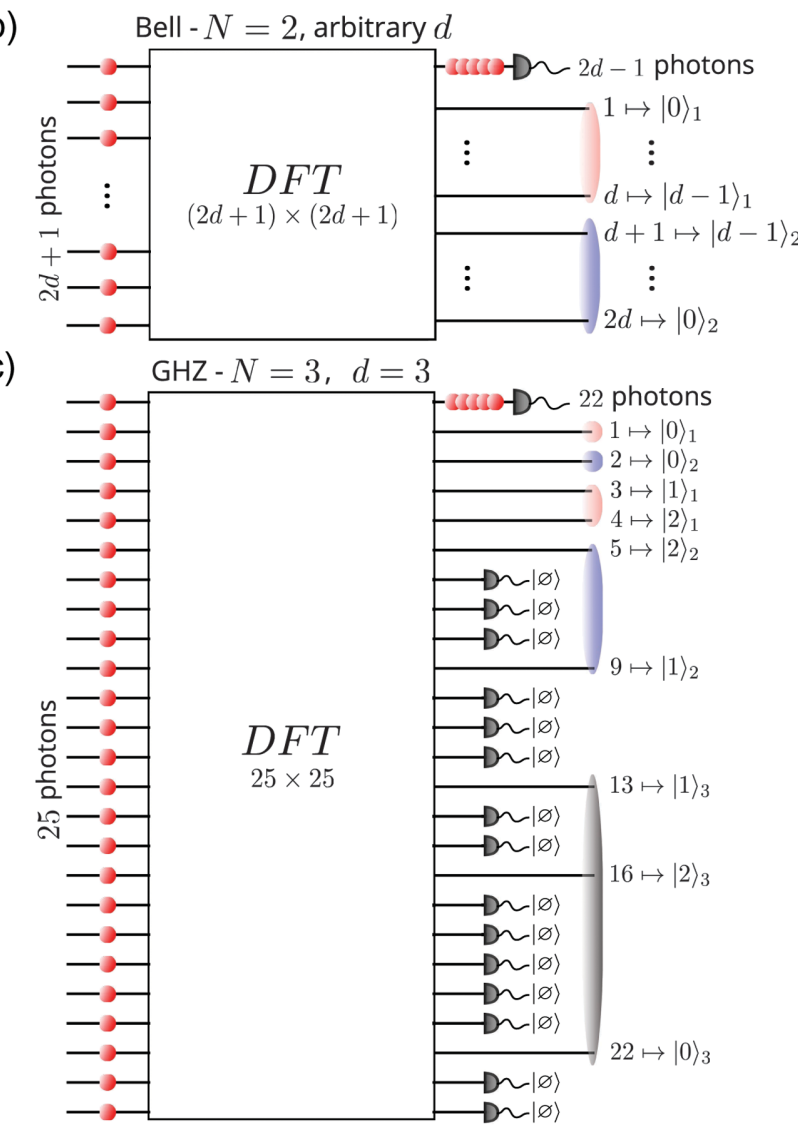

FIG. 1. (a) General schematic for the heralded generation of $N$-photon $d$-dimensional GHZ states via a DFT interferometer. The scheme uses the set of $N d$ modes $\mathcal{X}$ to encode the qudits and the complementary set of $m-N d$ modes $\overline{\mathcal{X}}$ are detected for heralding. It requires a single number resolving detector for heralding in the zero-th output mode, while the rest of modes in $\overline{\mathcal{X}}$ use threshold detectors heralding the vacuum. (b) Optimized solution for the heralded generation of Bell states $(N=2)$ in arbitrary dimension, requiring $m=2 d+1$. The correspondence between the optimal modes and the associated computational states of each qudit is shown. (c) Optimized solution for the heralded generation of three-photon three-dimensional GHZ states using the DFT scheme, requiring $m=25$. Output modes associated to the three different qudits are highlighted with different colors, and the associated qudit computational states are labeled.

$$
\sum_{i=1}^{m} c_{i} \bmod m=0
$$

The vector $\vec{c}$ has a number of elements given by the number of photons, here $m$. Each element $c_{i}$ labels the $i$ th photon's output mode, with modes indexed from 0 to $m-1$. For example, $\vec{c}=(0,1,1,4)$ represents the configuration with one photon in each of modes 0 and 4, two photons in mode 1 , and zero photons in all remaining modes. The ZTL condition is valid for any value of $m$.

To describe how the ZTL can be used to generate GHZ states, we introduce a notation for the encoding of individual qudits in the output modes of the DFT. Each qudit of dimension $d$ is encoded in a single photon propagating through $d$ optical modes (see, e.g., Refs. [7,8]). We will denote as $\mathcal{X} \subset\{0, \ldots, m-1\}$ the set of $N d$ modes used to encode the $N$ qudits, while its complement $\overline{\mathcal{X}}=\{0, \ldots, m-1\} \backslash \mathcal{X}$ (the remaining set of $m-N d$ modes) represents all the auxiliary modes used for heralding the desired state. Each of the $N$ qudits is defined via a set of $d$ distinct modes $Q_{i}=\left\{x_{0}^{(i)}, x_{1}^{(i)}, \ldots, x_{d-1}^{(i)}\right\} \subset \mathcal{X}$, with $x_{k}^{(i)} \neq x_{k^{\prime}}^{(i)}$ if $k \neq k^{\prime}$, so that all the qudits $\left\{Q_{i}\right\}$ form a partition of $\mathcal{X}$. We say that the $i$ th qudit is in the logical state $|k\rangle_{i}$ if a single photon is present in mode $x_{k}^{(i)}$, while all other modes in the set $Q_{i}$ are vacuum.

For simplicity, we will focus on heralding configurations where all the $m-N$ auxiliary photons are detected in the zeroth mode, and vacuum in the remaining heralding modes $\overline{\mathcal{X}} \backslash\{0\}$, as shown in Fig. 1(a). In this way, all auxiliary photons give zero contributions to the sum in Eq. (1), and only contributions from the $N$ encoding photons remain. Under this condition, we can write the set of all possible remaining $\mathrm{N}$-photon output states induced by the heralding on $\overline{\mathcal{X}}$ and the ZTL as

$$
\begin{aligned}
\mathcal{B}_{\mathcal{X}}= & \left\{\vec{b}_{k}=\left(y_{k, 1}, y_{k, 2}, \ldots, y_{k, N}\right) \mid\right. \\
& \left.\sum_{i=1}^{N} y_{k, i} \quad \bmod m=0, y_{k, i} \in \mathcal{X}\right\} .
\end{aligned}
$$

Here $\vec{b}_{k}$ are the output configurations allowed by the ZTL, indexed by $k$, and $y_{k, i}$ represent each photon's output port for that configuration, as in Eq. (1).

We will now show that the following conditions for the set $\mathcal{B}_{\mathcal{X}}$ are sufficient (but not necessary) to obtain a GHZ state at the output: (1) $\mathcal{B}_{\mathcal{X}}$ contains exactly $d$ configurations, i.e., $\left|\mathcal{B}_{\mathcal{X}}\right|=d$. (2) $\mathcal{B}_{\mathcal{X}}$ forms a partition of $\mathcal{X}$. (3) For all $\vec{b}_{k} \in \mathcal{B}_{\mathcal{X}}, \sum_{i=1}^{N} y_{k, i}=m$. This is a slightly more restrictive form of the ZTL. The first step is to show that, if these three conditions are satisfied, we can specify $N$ well-defined photonic qudits, given by 
$Q_{i}=\left\{x_{k}^{(i)}=y_{k, i}\right\}_{k=0, \ldots, d-1}$. Note that, because the sets $\left\{Q_{i}\right\}$ are given by a simple transposition of indices of $\mathcal{B}_{\mathcal{X}}$, condition 2 immediately implies that $\left\{Q_{i}\right\}$ also forms a partition of $\mathcal{X}$. Moreover, conditions 1 and 2 imply that all $y_{k, i}$ are different, i.e., $y_{k, i} \neq y_{k^{\prime}, i^{\prime}}$ if $k \neq k^{\prime}$ or $i \neq i^{\prime}$. In fact, because $\mathcal{B}_{\mathcal{X}}$ contains exactly $d$ configurations of $N$ elements, if any two $y_{k, i}$ were identical then necessarily $\left|\cup_{k} \vec{b}_{k}\right|<N d$, and thus $\mathcal{B}_{\mathcal{X}}$ could not form a partition of $\mathcal{X}$, given that $|\mathcal{X}|=N d$. Finally, from their definition and the fact that the elements $y_{k, i}$ are all different, it immediately follows that each set $Q_{i}$ contains exactly one output photon. Our specification for the sets $\left\{Q_{i}\right\}$ therefore defines $N$ disjoint sets of $d$ different modes, each set containing exactly one photon, and thus provides a valid encoding for the $N$ qudits. We are now left to verify that the state of the $N$ qudits is in fact a GHZ state.

Note that, with the definition used for the $Q_{i}$ 's, the $d$ elements $\vec{b}_{k}$ of $\mathcal{B}_{\mathcal{X}}$ correspond to the logical $N$-qudit states $\vec{b}_{k} \mapsto|k, k, \ldots, k\rangle, k \in 0, \ldots, d-1$. Because of the ZTL, the total output state is therefore a superposition of the states $|k, k, \ldots, k\rangle$. As shown in Supplemental Material 1 [27], condition 3 ensures that all amplitudes in such superposition are uniform and nonzero, thus providing the desired $N$-photon GHZ state in $d$ dimensions.

General and optimized schemes. - The task of generating GHZ states for a given photon number $N$ and dimension $d$ can now be reduced to a combinatorial number theory problem: finding an integer number $m$ and a set $\mathcal{X} \subseteq\{1,2, \ldots, m-1\}$ ( 0 is occupied for the heralding) so that $\mathcal{B}_{\mathcal{X}}$ satisfies conditions 1-3. Solutions can be found for any $N$ and $d$. An example of such general solutions is given by the set

$\mathcal{X}=\left\{N^{i-1}\right\}_{i \in[1,(N-1) d]} \cup\left\{m-\sum_{j=0}^{N-2} N^{j d+k}\right\}_{k \in[0, d-1]}$,

with $\quad m=\left(N^{N d}-1\right) /\left(N^{d}-1\right)$, with details in Supplemental Material 2 [27]. While this particular solution is highly suboptimal in the number of resources and success probability, and likely not suitable for practical implementations, it is general and shows that arbitrary $N$ photon $d$-dimensional GHZ states can, in principle, be generated with linear optics.

More efficient solutions can be found on a case-by-case basis. For example, in the simple case with $N=2$, i.e., heralded generation of qudit Bell pairs, a solution for any $d$ can easily be found by taking $m=2 d+1$ and $\mathcal{X}=\{1,2, \ldots, 2 d\}$. This gives $\mathcal{B}_{\mathcal{X}}=\{(1,2 d),(2,2 d-$ $1), \ldots,(d, d+1)\}$ and qudit modes $Q_{1}=\{1,2 \ldots, d\}$, $Q_{2}=\{2 d, 2 d-1 \ldots, d+1\}$, as shown in Fig. 1(b). The success probability for the state generation is given by $d(2 d-1) ! /(2 d+1)^{2 d-1}$ (see Supplemental Material 1 and 6 for additional schemes for qudit Bell states [27]).
For small values of $N$ and $d$, optimized solutions for our heralded GHZ generation scheme can be found numerically. For example, in Fig. 1(c) we report the optimized solution for the case of heralded GHZ generation for $(N, d)=(3,3)$, which requires $m=25$. The list of modes used for the encoding in this case is $\mathcal{X}=\{1,2,3,4,5,9,13,16,22\}$, and the only threeelement combinations (including repetitions) that sum up to multiples of $m$, given by $\mathcal{B}_{\mathcal{X}}=\{(1,2,22)$, $(3,9,13),(4,5,16)\}$, satisfy conditions $1-3$. This therefore provides heralded GHZ generation for the three qudits defined in the modes $Q_{1}=\{1,3,4\}, Q_{2}=\{2,9,5\}$, $Q_{3}=\{22,13,16\}$. Note that, at the output of the DFT, adjacent modes associated to different qudits can be interleaved; in such cases, a network of swaps is required to separate the different qudits and address them individually. However, without further optimization, the threedimensional GHZ generation success probability remains very small, approximately $10^{-10}$.

The main reason for low success probabilities is the use of a single heralding pattern among exponentially many possible outcomes. While the choice of a single heralding pattern was done to simplify the treatment in the general case of arbitrary $N$ and $d$, for a given $N$ and $d$ many more heralding patterns are likely to generate GHZ entanglement. We show in Supplemental Material 3 [27] that these (combinatorially many) valid heralding patterns can be used, in conjunction with feed-forward operations and balancing circuitry, to significantly improve the success probability. For example, Monte Carlo simulations of the $(N, d)=(3,3)$ GHZ generation scheme of Fig. 1(c) show that the success probability is boosted to approximately $10^{-4}$. This indicates that many orders of magnitude improvements can be found through solution-specific optimizations. We estimate that, with this optimization, heralded $(3,3)$ GHZ generation could be achieved at $0.7 \mathrm{kHz}$ rates with state-of-the-art quantum photonic hardware. Furthermore, in Supplemental Material 2 [27] we report an algorithm to estimate optimized solutions for larger values of $N$ and $d$.

Constructing universal cluster states of qudits.-The DFT-based schemes described above can be generalized to generate a large class of $\mathrm{N}$-photon states with dimensionality $d$, but at the cost of exponentially large resources when increasing $N$ (see Appendix 4 in the Supplemental Material [27]). However, due to the heralded nature of the generated high-dimensional entanglement, we can use these schemes to adapt qubit-based LOQC techniques (e.g., multiplexing and fusion operations [5,46]) to construct a highdimensional architecture that is scalable. Measurementbased quantum computing (MBQC) [47] in linear optics typically proceeds by connecting small entangled resource states using probabilistic fusion gates [24], to build large cluster states. Similarly, high-dimensional photonic GHZ states can be used as building blocks to construct large 


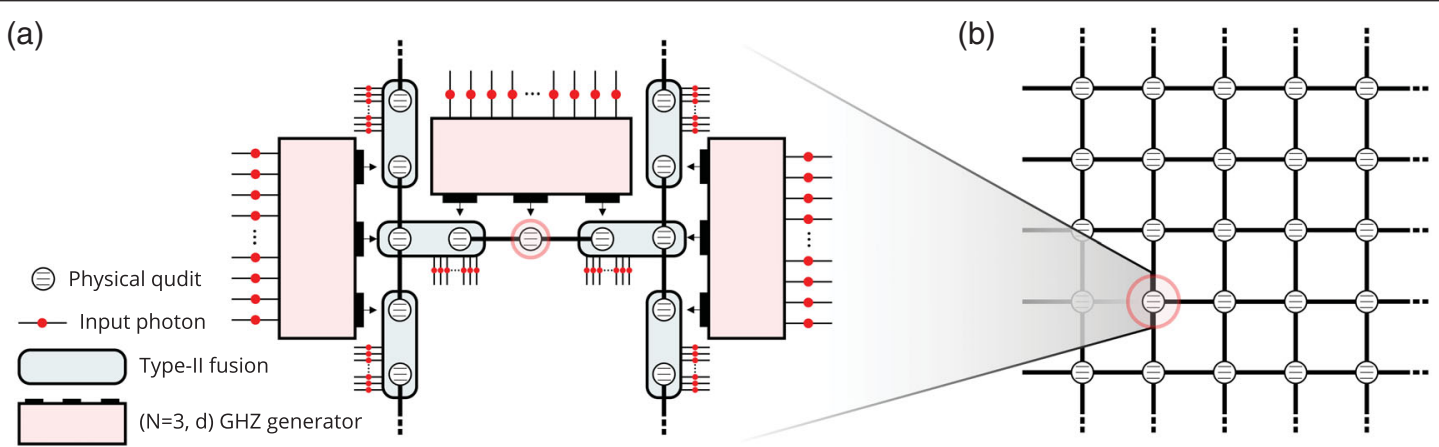

FIG. 2. Example of modular architecture for constructing universal cluster states from three-photon GHZ states with linear optics. Each module, shown in (a), arranges three-photon GHZ state generators and fusion gates such that a single $d$-dimensional qudit is linked to the four neighboring qudits in the universal square lattice in (b).

high-dimensional cluster states for MBQC. Two recent protocols for type-II fusion (destructive Bell state measurements) of arbitrary dimensional qudits have been proposed independently: Luo et al. [13] use unentangled single photons and $d-2$ ancillary photons; Zhang et al. [48] use $d-1$ Bell states. The success probability of both fusion gates scales $\approx 1 / d^{2}$.

As shown in Fig. 2, we can combine type-II highdimensional fusion operations with three-photon highdimensional GHZ generators in a modular approach to build up universal cluster states of qudits. The square lattice of qudits shown in Fig. 2(b) is an example of a cluster state universal for high-dimensional MBQC [49,50]. Figure 2(a) shows a module for the architecture, where multiple GHZ states are fused together to link a single computational photonic qudit to the rest of the lattice. Once such states are built, universal measurement-based high-dimensional quantum computing can be performed [47,49,50]: operations on the logical qudits encoded in the rows of the lattice are performed via measurement and feed-forward, with the output state encoded in the qudits of the last layer of the lattice. Because the resource is universal, any pure high-dimensional multiphoton state can be prepared as a result of the computation up to arbitrary precision.

On the other hand, because both the GHZ generation and the type-II fusion gates have low success probability, the total probability to successfully generate high-dimensional qudit cluster states can in general vanish quite rapidly when increasing the number of qudits or the dimensionality. Nevertheless, by adapting techniques already developed for qubit-based loss-tolerant LOQC architectures, the approach can, in principle, be made scalable and the total success probability boosted to near unity $[24,46,51,52]$. For example, because the GHZ generation is heralded, gate multiplexing can be used to render the production of GHZ states near deterministic with a resource overhead that scales approximately linearly with the generation success probability [46,53]. Repeat-until-success proposals provide a flexible approach to correct for the limited success probability of the fusion gates, at the cost of requiring quantum memories [24]. Moreover, if the fusion success probability is improved above the percolation threshold of the lattice used, ballistic architectures can be used to correct the unsuccessful fusion gates directly on the generated lattice without the use of quantum memories [46,51]. However, this approach would likely require the use of lattices with increased valency to bring the percolation threshold above the current qudit fusion gate's success rates [54]. In addition, it may be possible to increase the success probability of the qudit fusion gates by using additional ancilla resources, as has been shown for qubits $[55,56]$.

Robustness against photon distinguishability.-While requiring additional resources compared to qubit-based approaches, qudits can provide improved robustness to noise, with potential benefits for quantum communication and fault-tolerant quantum technologies [57-60]. Here, we numerically analyze how distinguishability, an important source of noise in quantum photonics, affects the multiphoton high-dimensional entanglement generated using the schemes proposed in Fig 1. We assume that all photons have pure internal states such that they all share the same value of pairwise indistinguishability $\left|\left\langle\psi_{i} \mid \psi_{j}\right\rangle\right|^{2}$. Thus all pairs of photons would exhibit the same Hong-Ou-Mandel interference visibility. To determine the effect of distinguishability, we numerically reconstruct the simulated output heralded state within the qudit space for different values of indistinguishability $\left|\left\langle\psi_{i} \mid \psi_{j}\right\rangle\right|^{2}$. The noisy state generation is simulated using an approach by Tichy describing multiphoton interference of partially distinguishable photons [61] (see Supplemental Material 7 for details [27]). Because the number of photons involved in the scheme increases rapidly with $N$ and $d$, this simulation quickly becomes intractable when increasing the heralded state complexity. Nevertheless, for the scheme shown in Fig. 1(b) with $N=2$ and low values of $d$, the simulation remains tractable on a standard laptop.

For various levels of distinguishability, we show in Fig. 3(a) the fidelity of the generated state to the ideal qudit Bell state, and in Fig. 3(b) the logarithmic negativity as a figure of merit to assess the generated entanglement. 

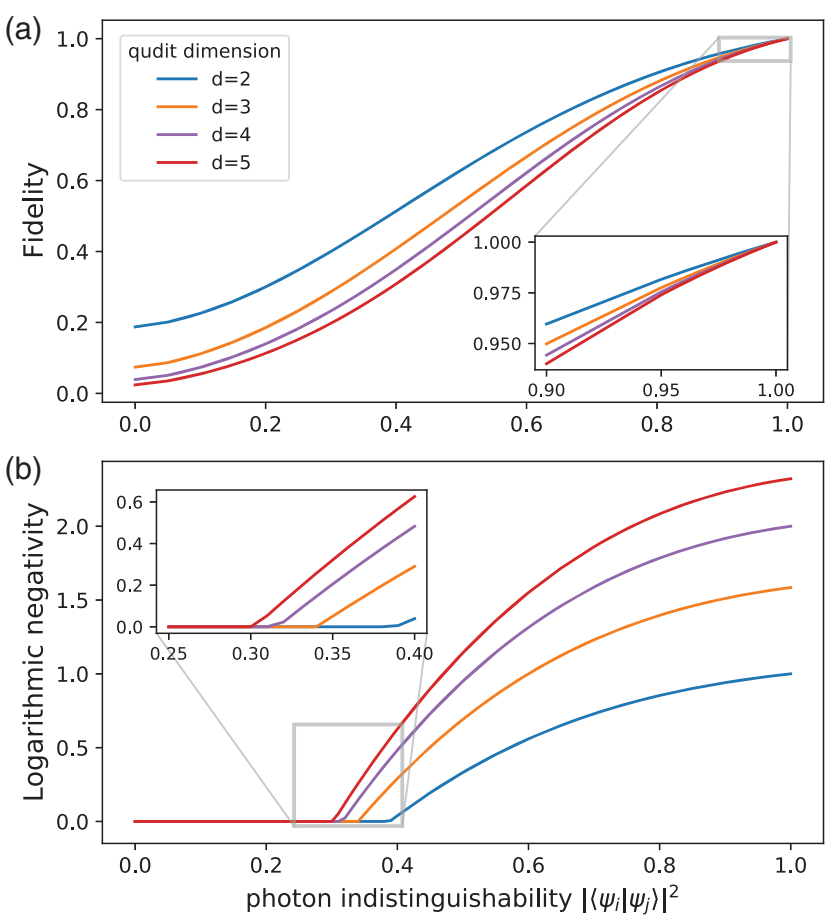

FIG. 3. Robustness of the qudit Bell state generation circuits against photon indistinguishability. (a) Quantum fidelity of the generated qudit Bell state with the ideal, plotted against photon indistinguishability. (b) The logarithmic negativity bound on the distillable entanglement from the generated state with photon indistinguishability.

The logarithmic negativity is an entanglement monotone that upper bounds the distillable entanglement, which in turn quantifies the amount of pure state entanglement that can be extracted under local operations and classical communication [62,63]. Distinguishability weakens the interference governing the ZTL, and therefore both the state fidelity and generated entanglement decrease when the indistinguishability is reduced. However, while the fidelity is always lower for higher dimensional states, the negativity reaches zero at larger values of photon distinguishability for higher dimensions, as highlighted in the inset of Fig. 3(b). This indicates that for increased dimensionality, the generated entanglement can endure higher levels of photon distinguishability, even if the number of imperfect input photons is larger.

Discussion.-The large resource overheads of highdimensional LOQC could be compensated to some extent by quantum error correction protocols that are more efficient due to robustness to noise that increases with qudit dimension. Furthermore, and similarly to the improvements made in efficiency for qubit-based LOQC $[23,24,46]$, we expect our results to provide a first and important step for developing high-dimensional LOQC architectures closer to midterm technological capabilities. For example, as detailed in Supplemental Material 3 [27], by considering all valid heralding events in our GHZ generation scheme for the particular case with $d=3$ [see Fig. 1(c)], the resource overheads for multiplexing are reduced from $\mathcal{O}\left(10^{10}\right)$ to $\mathcal{O}\left(10^{4}\right)$, showing that enormous improvements are possible via solution-specific optimization. Crucially, this work proves that such solutions can exist for any dimension.

We thank C. Vigliar, D. Bacco, M. Erhard and M. Krenn for useful discussions. We acknowledge support from the Engineering and Physical Sciences Research Council (EPSRC) Hub in Quantum Computing and Simulation (EP/T001062/1) and Networked Quantum Information Technologies (EP/N509711/1). Fellowship support from EPSRC is acknowledged by A. L. (EP/N003470/1).

*stefano.paesani@nbi.ku.dk

[1] P. Kok, W. J. Munro, K. Nemoto, T. C. Ralph, J. P. Dowling, and G. J. Milburn, Rev. Mod. Phys. 79, 135 (2007).

[2] J. L. O'Brien, A. Furusawa, and J. Vučković, Nat. Photonics 3, 687 (2009).

[3] J. Yin, Y. Cao, Y.-H. Li, S.-K. Liao, L. Zhang, J.-G. Ren, W.-Q. Cai, W.-Y. Liu, B. Li, H. Dai et al., Science 356, 1140 (2017).

[4] H. Wang, J. Qin, X. Ding, M.-C. Chen, S. Chen, X. You, Y.-M. He, X. Jiang, L. You, Z. Wang, C. Schneider, J. J. Renema, S. Höfling, C.-Y. Lu, and J.-W. Pan, Phys. Rev. Lett. 123, 250503 (2019).

[5] T. Rudolph, APL Photonics 2, 030901 (2017).

[6] M. Erhard, M. Krenn, and A. Zeilinger, Nat. Rev. Phys. 2, 365 (2020).

[7] J. Wang, S. Paesani, Y. Ding, R. Santagati, P. Skrzypczyk, A. Salavrakos, J. Tura, R. Augusiak, L. Mančinska, D. Bacco et al., Science 360, 285 (2018).

[8] C. Vigliar, S. Paesani, Y. Ding, J. Adcock, J. Wang, S. Morley-Short, D. Bacco, L. Oxenløwe, M. Thompson, J. Rarity, and A. Laing, arXiv:2005.06443.

[9] E. Gómez, S. Gómez, I. Machuca, A. Cabello, S. Pádua, S. Walborn, and G. Lima, Phys. Rev. Applied 15, 034024 (2021).

[10] A. C. Dada, J. Leach, G. S. Buller, M. J. Padgett, and E. Andersson, Nat. Phys. 7, 677 (2011).

[11] R. Fickler, R. Lapkiewicz, W. N. Plick, M. Krenn, C. Schaeff, S. Ramelow, and A. Zeilinger, Science 338, 640 (2012).

[12] M. Malik, M. Erhard, M. Huber, M. Krenn, R. Fickler, and A. Zeilinger, Nat. Photonics 10, 248 (2016).

[13] Y.-H. Luo, H.-S. Zhong, M. Erhard, X.-L. Wang, L.-C. Peng, M. Krenn, X. Jiang, L. Li, N.-L. Liu, C.-Y. Lu, A. Zeilinger, and J.-W. Pan, Phys. Rev. Lett. 123, 070505 (2019).

[14] M. Erhard, M. Malik, M. Krenn, and A. Zeilinger, Nat. Photonics 12, 759 (2018).

[15] M. Kues, C. Reimer, P. Roztocki, L. R. Cortés, S. Sciara, B. Wetzel, Y. Zhang, A. Cino, S. T. Chu, B. E. Little et al., Nature (London) 546, 622 (2017).

[16] C. Reimer, S. Sciara, P. Roztocki, M. Islam, L. R. Cortés, Y. Zhang, B. Fischer, S. Loranger, R. Kashyap, A. Cino et al., Nat. Phys. 15, 148 (2019). 
[17] C. Lee, D. Bunandar, Z. Zhang, G. R. Steinbrecher, P. B. Dixon, F. N. Wong, J. H. Shapiro, S. A. Hamilton, and D. Englund, arXiv:1611.01139.

[18] A. Martin, T. Guerreiro, A. Tiranov, S. Designolle, F. Fröwis, N. Brunner, M. Huber, and N. Gisin, Phys. Rev. Lett. 118, 110501 (2017).

[19] J. Carolan, C. Harrold, C. Sparrow, E. Martín-López, N. J. Russell, J. W. Silverstone, P. J. Shadbolt, N. Matsuda, M. Oguma, M. Itoh et al., Science 349, 711 (2015).

[20] J. Cariñe, G. Cañas, P. Skrzypczyk, I. Šupić, N. Guerrero, T. Garcia, L. Pereira, M. Prosser, G. B. Xavier, A. Delgado et al., Optica 7, 542 (2020).

[21] X. Gu, M. Erhard, A. Zeilinger, and M. Krenn, Proc. Natl. Acad. Sci. U.S.A. 116, 4147 (2019).

[22] M. Krenn, J. Kottmann, N. Tischler, and A. Aspuru-Guzik, arXiv:2005.06443.

[23] E. Knill, R. Laflamme, and G. J. Milburn, Nature (London) 409, 46 (2001).

[24] D. E. Browne and T. Rudolph, Phys. Rev. Lett. 95, 010501 (2005).

[25] M. C. Tichy, K. Mayer, A. Buchleitner, and K. Mølmer, Phys. Rev. Lett. 113, 020502 (2014).

[26] A. Crespi, R. Osellame, R. Ramponi, M. Bentivegna, F. Flamini, N. Spagnolo, N. Viggianiello, L. Innocenti, P. Mataloni, and F. Sciarrino, Nat. Commun. 7, 10469 (2016).

[27] See Supplemental Material at http://link.aps.org/ supplemental/10.1103/PhysRevLett.126.230504 for details of the linear optical schemes developed and methods for their simulation and optimization, which includes Refs. [28-45].

[28] M. C. Tichy, M. Tiersch, F. de Melo, F. Mintert, and A. Buchleitner, Phys. Rev. Lett. 104, 220405 (2010).

[29] L. Babel, H. Kellerer, and V. Kotov, Math. Methods Oper. Res. 47, 59 (1998).

[30] P. Erdös, J. Lond. Wash. Soc. 16, 212 (1941).

[31] R. K. Guy, in North-Holland Mathematics Studies, Vol. 60 (Elsevier, New York, 1982), pp. 141-154.

[32] T. Bohman, Electron. J. Comb. 5, R3 (1998).

[33] https://github.com/jakeffbulmer/lo_sim/.

[34] J. Joo, P. L. Knight, J. L. O'Brien, and T. Rudolph, Phys. Rev. A 76, 052326 (2007).

[35] P. Clifford and R. Clifford, in Proceedings of the Twenty-Ninth Annual ACM-SIAM Symposium on Discrete Algorithms (SIAM, 2018), pp. 146-155.

[36] R. Uppu, F. T. Pedersen, Y. Wang, C. T. Olesen, C. Papon, X. Zhou, L. Midolo, S. Scholz, A. D. Wieck, A. Ludwig, and P. Lodahl, Sci. Adv. 6, eabc8268 (2020).

[37] H.-S. Zhong, H. Wang, Y.-H. Deng, M.-C. Chen, L.-C. Peng, Y.-H. Luo, J. Qin, D. Wu, and X. Ding et al., Science 370, 1460 (2020).
[38] D. Fukuda, G. Fujii, T. Numata, K. Amemiya, A. Yoshizawa, H. Tsuchida, H. Fujino, H. Ishii, T. Itatani, S. Inoue, and T. Zama, Opt. Express 19, 870 (2011).

[39] M. Huber and J. I. de Vicente, Phys. Rev. Lett. 110, 030501 (2013).

[40] J. C. Adcock, S. Morley-Short, J. W. Silverstone, and M. G. Thompson, Quantum Sci. Technol. 4, 015010 (2018).

[41] M. Krenn, X. Gu, and A. Zeilinger, Phys. Rev. Lett. 119, 240403 (2017).

[42] Q. Zhang, X.-H. Bao, C.-Y. Lu, X.-Q. Zhou, T. Yang, T. Rudolph, and J.-W. Pan, Phys. Rev. A 77, 062316 (2008).

[43] X. B. Zou, K. Pahlke, and W. Mathis, J. Opt. B 7, 119 (2005).

[44] C. M. Caves and G. J. Milburn, Opt. Commun. 179, 439 (2000).

[45] R. T. Thew, K. Nemoto, A. G. White, and W. J. Munro, Phys. Rev. A 66, 012303 (2002).

[46] M. Gimeno-Segovia, P. Shadbolt, D. E. Browne, and T. Rudolph, Phys. Rev. Lett. 115, 020502 (2015).

[47] R. Raussendorf and H. J. Briegel, Phys. Rev. Lett. 86, 5188 (2001).

[48] C. Zhang, J. F. Chen, C. Cui, J. P. Dowling, Z. Y. Ou, and T. Byrnes, Phys. Rev. A 100, 032330 (2019).

[49] D. L. Zhou, B. Zeng, Z. Xu, and C. P. Sun, Phys. Rev. A 68, 062303 (2003).

[50] W. Hall, arXiv:quant-ph/0512130.

[51] K. Kieling, T. Rudolph, and J. Eisert, Phys. Rev. Lett. 99, 130501 (2007).

[52] Y. Li, P. C. Humphreys, G. J. Mendoza, and S. C. Benjamin, Phys. Rev. X 5, 041007 (2015).

[53] M. Gimeno-Segovia, H. Cable, G. J. Mendoza, P. Shadbolt, J. W. Silverstone, J. Carolan, M. G. Thompson, J. L. O'Brien, and T. Rudolph, New J. Phys. 19, 063013 (2017).

[54] S. Galam and A. Mauger, Phys. Rev. E 53, 2177 (1996).

[55] W. P. Grice, Phys. Rev. A 84, 042331 (2011).

[56] F. Ewert and P. van Loock, Phys. Rev. Lett. 113, 140403 (2014).

[57] L. Sheridan and V. Scarani, Phys. Rev. A 82, 030301(R) (2010).

[58] E. T. Campbell, Phys. Rev. Lett. 113, 230501 (2014).

[59] F. H. E. Watson, H. Anwar, and D. E. Browne, Phys. Rev. A 92, 032309 (2015).

[60] S. Ecker, F. Bouchard, L. Bulla, F. Brandt, O. Kohout, F. Steinlechner, R. Fickler, M. Malik, Y. Guryanova, R. Ursin, and M. Huber, Phys. Rev. X 9, 041042 (2019).

[61] M. C. Tichy, Phys. Rev. A 91, 022316 (2015).

[62] G. Vidal and R.F. Werner, Phys. Rev. A 65, 032314 (2002).

[63] M. B. Plenio, Phys. Rev. Lett. 95, 090503 (2005). 